\title{
zVAD-fmk, unlike BocD-fmk, does not inhibit caspase-6 acting on 14-3-3/Bad pathway in apoptosis of p815 mastocytoma cells
}

\author{
Su-Bog Yee ${ }^{1 *}$, Soo Jin Baek ${ }^{1,2 *}$, \\ Hwan Tae Park', Seung Hun Jeong ${ }^{1}$, \\ Jin Hee Jeong ${ }^{1}$, Tae Hyun $\mathrm{Kim}^{1}$, \\ Jong-Min Kim ${ }^{1}$, Byung Kap Jeong ${ }^{3}$, \\ Bong Soo Park', Taeg Kyu Kwon ${ }^{5}$, \\ II Yoon ${ }^{4}$ and Young Hyun Yoo ${ }^{1,6}$
}

\author{
${ }^{1}$ Department of Anatomy and Cell Biology (BK21 program) \\ ${ }^{2}$ Department of Physiology \\ Dong-A University College of Medicine and \\ Medical Science Research Center \\ Busan 602-714, Korea \\ ${ }^{3}$ Department of Biological Sciences \\ Kosin University \\ Busan 606-701, Korea \\ ${ }^{4}$ College of Dentistry and \\ Research Institute for Oral Biotechnology \\ Pusan National University \\ Busan 602-739, Korea \\ ${ }^{5}$ Department of Immunology \\ School of Medicine, Keimyung University \\ Daegu 704-701, Korea \\ ${ }^{6}$ Corresponding author: Tel, 82-51-240-2926; \\ Fax, 82-51-241-3767; E-mail, yhyoo@dau.ac.kr \\ ${ }^{*}$ These authors contributed equally to this work.
}

\section{Accepted 20 October 2006}

Abbreviations: Ac-DEVD-afc, acetyl-Asp-Glu-Val-Asp-7-amido-4-trifluoromethylcoumarin; Ac-IETD-afc, acetyl-lle-Glu-Thr-Asp-7-amido-4-trifluoromethylcoumarin; Ac-LEHD-afc, acetyl-Leu-Glu-HisAsp-7-amido-4-trifluoromethylcoumarin; Ac-VDVAD-afc, acetyl-ValAsp-Val-Ala-Asp-7-amido-4-trifluoromethylcoumarin; Ac-VEID-afc, acetyl-Val-Glu-Ile-Asp-7-amido-4-trifluoromethylcoumarin; Ac-WEHD-afc, acetyl-Trp-Glu-His-Asp-7-amido-4-trifluoromethylcoumarin; Ac-YVAD-afc, acetyl-Tyr-Val-Ala-Asp-7-amido-4-trifluoromethylcoumarin; BocD-fmk, Boc-aspartyl(OMe)-fluoromethylketone; DIC, differential interference contrast; z-DEVD-fmk, benzyloxycarbonyl-Asp(OMe)-Glu(OMe)-Val-Asp(OMe)-fluoromethylketone; zVAD-fmk, benzyloxycarbonyl-Val-Ala-Asp(OMe)-fluoromethylketone; z-VDVAD-fmk, benzyloxy-carbonyl-Val-Asp(OMe)-Val-Ala-Asp(OMe)fluoromethylketone; z-VEID-fmk, benzyloxycarbonyl-Val-Glu(OMe)lle-Asp(OMe)-fluoromethylketone

\section{Abstract \\ In a preliminary study, we found that benzyloxycar-}

bonyl-Val-Ala-Asp(OMe)-fluoromethylketone (zVADfmk), unlike Boc-aspartyl(OMe)-fluoromethylketone (BocD-fmk), at usual dosage could not prevent genistein-induced apoptosis of p815 mastocytoma cells. This study was undertaken to reveal the mechanism underlying the incapability of ZVAD-fmk in preventing this type of apoptosis. We observed that 14-3-3 protein level was reduced in genistein-treated cells and that BocD-fmk but not ZVAD-fmk prevented the reduction of 14-3-3 protein level and the release of Bad from 14-3-3. We also demonstrated that truncated $\mathrm{Bad}$ to $\mathrm{Bcl}-\mathrm{xL}$ interaction in genisteintreated cells was prevented by BocD-fmk but not by zVAD-fmk treatment. Our data indicate that BocDfmk, compared to zVAD-fmk, has a certain preference for inhibiting 14-3-3/Bad signalling pathway. We also elucidated that this differential efficacy of BocD-fmk and zVAD-fmk resulted from the different effect in inhibiting caspase- 6 and that co-treatment of zVAD-fmk and caspase- 6 specific inhibitor substantially prevented genistein-induced apoptosis. Our data shows that caspase- 6 plays a role on $\mathrm{Bad} / 14-3-3$ pathway in genistein-induced apoptosis of $p 815$ cells, and that the usual dose of zVAD-fmk, in contrast to BocD-fmk, did not prevent caspase-6 acting on 14-3-3/Bad-mediated event.

Keywords: $14-3-3$ proteins; benzyloxycarbonylvalylalanyl-aspartyl fluoromethyl ketone; bcl-associated death protein; caspase 6; genistein; mastocytoma

\section{Introduction}

Apoptosis, or programmed cell death, is an essential physiological process that is required for the normal development and maintenance of tissue homeostasis. However, apoptosis also is implicated in a wide range of pathological conditions, including immunological diseases, allergy and cancer (Holtzman et al., 2000; Zimmermann et al., 2001; Gong et al., 2004; Du et al., 2006). During apoptosis, cells undergo specific morphological and biochemical changes, including cell shrinkage, chromatin condensation, and internucleosomal cleavage of genomic DNA (Reed, 2001; Kankaanranta et al., 2002). Apoptosis has been mainly studied in inflammatory cells such as eosinophils, neutrophils, lymphocytes and macrophages that participate in the late and chronic stages 
of allergy as a new way to elucidate the pathogenesis of this disease, although much less is known about the regulation of mast cell apoptosis in the immediatephase allergic process (Piliponsky et al., 2000).

Although tissue mast cell numbers under normal conditions are relatively constant from individual to individual, mast cells must proliferate rapidly and become activated in various pathological conditions and processes, including mastocytosis, chronic inflammatory conditions, or allergic diseases. The number of mast cells in tissue was demonstrated to be regulated in part by apoptosis (Metcalfe et al., 1995; Park et al., 2000).

Pharmacological inhibitors are a convenient method to study the activity of enzymes. Numerous studies on the physiological functions of caspases and the regulation of their activity have been being conducted by employing caspase inhibitor(s). Thus much of our understanding of caspases is due to the use of these inhibitors. Currently, each caspase subtype inhibitors and broad-caspase inhibitors are commercially available.

zVAD-fmk and BocD-fmk are widely used cell permeable broad-caspase inhibitors. These broadcaspase inhibitors, in general, prevent apoptosis by inhibiting the activation of all caspase subtypes. However, these broad-caspase inhibitors could not prevent all types of apoptosis. The incapability of broad-caspase inhibitors in preventing apoptosis might result from the presence of a certain apoptotic factor insensitive to caspase inhibitors (Chipuk et al., 2005). In some apoptosis systems, these inhibitors could not prevent partial, mostly cytoplasmic, features of apoptosis (McCarthy et al., 1997). Otherwise, the incapability of these inhibitors in preventing apoptosis might be due to the fact that these inhibitors do not inhibit all caspases to the same extent. For example, caspase-2 has been reported to be relatively resistant to zVAD-fmk since approximately 1,000-fold more zVAD-fmk was required to inhibit caspase-2 compared with caspase-1 (Ekert et al., 1999).

While we had been undertaking the study on genistein-induced apoptosis in p815 mastocytoma cells (Park et al., 2002), we observed that zVAD-fmk could not prevent the apoptosis (unpublished data). Thus, we undertook this study to unveil the mechanism underlying the incapability of zVAD-fmk in preventing apoptosis of p815 cells.

\section{Materials and Methods}

\section{Reagents}

The following antibodies were obtained commercially; rabbit polyclonal anti-horse cytochrome $c$, anti-mouse Bax and AIF, anti-human Bcl-2, Bcl-xL,
Bak, 14-3-3 $\beta$, CAD and Hsp60, mouse polyclonal anti-human Bad antibodies were from Santa Cruz Biotechnology (Santa Cruz, CA); rabbit polyclonal anti-human caspase- 6 was from Cell Signaling (Beverly, MA); rabbit polyclonal anti-mouse Smac/ DIABLO, phospho-Bad $\left(\mathrm{Ser}^{112}\right)$, phospho-Bad $\left(\operatorname{Ser}^{136}\right)$, and phospho-Bad $\left(\mathrm{Ser}^{155}\right)$ antibodies were from Calbiochem (San Diego, CA); monoclonal antiGAPDH (glyceraldehyde-3-phosphate dehydrogenase) antibody was from Chemicon (Temecula, CA); rabbit polyclonal anti-mouse Bid antibody was from Pharmingen (San Diego, CA); FITC-conjugated goat anti-rabbit IgG antibodies were from Vector (Burlingame, CA). Genistein was from Sigma (St. Louis, $\mathrm{MO})$; staurosporin, lactacystin, and caspase substrates and inhibitors were from Calbiochem (San Diego, CA); etoposide was from Dong-A Pharmaceutical Co., Ltd (Seoul, South Korea).

\section{Cell culture}

The p815 murine mastocytoma cells and rat basophilic leukemia RBL-2H3 cells were purchased from the American Type Culture Collection (Rockville, MD). P815 cells were maintained at $37^{\circ} \mathrm{C}$ with $5 \%$ $\mathrm{CO}_{2}$ in air atmosphere in Dulbecco's modified Eagle's medium with $4 \mathrm{mM}$ L-glutamine, $1.5 \mathrm{~g} / \mathrm{l}$ sodium bicarbonate, $4.5 \mathrm{~g} / \mathrm{l}$ glucose, and $1.0 \mathrm{mM}$ sodium pyruvate supplemented with $10 \%$ fetal bovine serum. RBL-2H cells were cultured in RPMI1640 medium with $10 \%$ fetal calf serum.

\section{Genistein treatment}

Since genistein at $50 \mu \mathrm{M}$ showed the half-maximal inhibition of viability, this single concentration was utilized to assess the efficacy of caspase inhibitors.

\section{Administration of caspase inhibitors}

Since usual dose of caspase inhibitors is $50 \mu \mathrm{M}$, cells treated with genistein were incubated with this dose of broad-caspase inhibitors for $16 \mathrm{~h}$. To analyze which caspase subtype is not blocked by zVAD-fmk in these genistein-treated cells, caspase$6,3,2$ caspase subtype inhibitors, z-VEID-fmk, z-DEVD-fmk, z-VDVAD-fmk at $50 \mu \mathrm{M}$ were administrated with $50 \mu \mathrm{M}$ zVAD-fmk.

\section{Trypan blue staining and Hoechst staining.}

Cells were harvested, washed with phosphate buffered saline (PBS), and stained with trypan blue and then counted using a hemacytometer. And cells and cell suspension were centrifuged, and stained in $4 \mu \mathrm{g} / \mathrm{ml}$ Hoechst 33342 and fixed in $4 \%$ paraformaldehyde. 


\section{Photomicrography and cell counting}

Total cell number, at least 300 cells from each experiment, was counted under DIC optics and the cell showing condensed or fragmented nuclei in Hoechst staining was calculated under epifluorescence optics by a blind observer.

\section{Western blot analysis}

Proteins from cells were separated in $10 \%$ SDSPAGE gels, transferred to nitrocellulose, and reacted with each antibody. Immunostaining was performed using SuperSignal West Pico enhanced chemiluminescence substrate (Pierce, Rockford, IL) and detected with LAS-3000PLUS (Fuji Photo Film Company, Kanagawa, Japan).

\section{Confocal microscopy}

Cells were cytocentrifuged and fixed in $4 \%$ paraformaldehyde, incubated with primary and FITCconjugated secondary antibodies, orderly. Fluorescent images were observed under Zeiss LSM 510 laser-scanning confocal microscope (Göttingen, Germany).

\section{Immunoprecipitation}

Cell extracts were incubated with antibodies in extraction buffer at $4^{\circ} \mathrm{C}$ overnight. Immunoprecipitated proteins or aliquots containing $500 \mu \mathrm{g}-1 \mathrm{mg}$ protein were separated on SDS-polyacrylamide gels, and Western blot analysis was performed as described above.

\section{Caspase subtype activity assay}

Caspase substrates, Ac-YVAD-afc, Ac-VDVAD-afc, Ac-DEVD-afc, Ac-WEHD-afc, Ac-VEID-afc, Ac-IETDafc, Ac-LEHD-afc were used for measuring the activity of caspase-1, 2, 3, 5, 6, 8, and 9. Caspase activities were assayed by incubating $30 \mu \mathrm{g}$ of cell lysates with $8 \mu$ of reaction buffer containing $15 \mu \mathrm{M}$ of each caspase substrate. Release of AFC was measured after $1 \mathrm{~h}$ of incubation at $37^{\circ} \mathrm{C}$ on a microplate fluorometer with excitation and emission wavelengths of 360 and $530 \mathrm{~nm}$.

\section{Statistical analysis}

Four independent experiments were carried out. The mean values were calculated from data obtained from triplicates of each independent experiment. The results were tested for statistical significance by the Kruskal-Wallis nonparametric test. $P$ value less than 0.05 were considered significant.

\section{Results}

z-VAD-fmk, unlike Boc-D-fmk, was unable to protect genistein-induced apoptosis in p815 cells

As determined by nuclear morphology and DNA electrophoresis, BocD-fmk at $50 \mu \mathrm{M}$ prevented genistein-induced apoptosis of p815 cells. However, zVAD-fmk at the same dose could not prevent the same type of apoptosis (Figure $1 \mathrm{~A}$ and $\mathrm{B}$ ). To know whether this phenomenon is stimulus- or cellselective, we treated p815 cells with other apoptosisinducing chemicals such as etoposide, staurosporin, and lactacystin. The results demonstrated that zVAD-fmk could not prevent apoptosis of p815 cells induced by all these chemicals. BocD-fmk, however, prevented these chemicals-induced apoptosis in p815 cells. On the other hand, zVAD-fmk as well as BocD-fmk prevented genistein-induced apoptosis in another mast cell line RBL-2H3 cells (Figure 2A). As determined by nuclear morphology and DNA electrophoresis, zVAD-fmk as well as BocD-fmk at 50 $\mu \mathrm{M}$ prevented genistein-induced apoptosis of RBL-
A

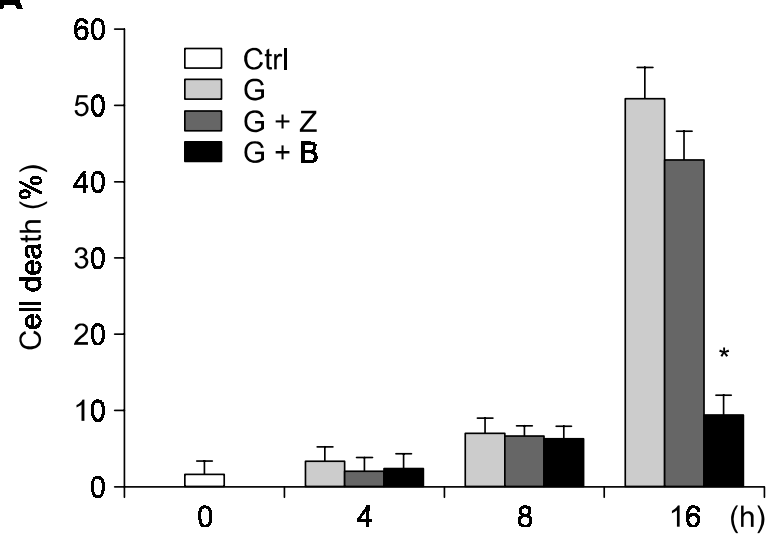

B

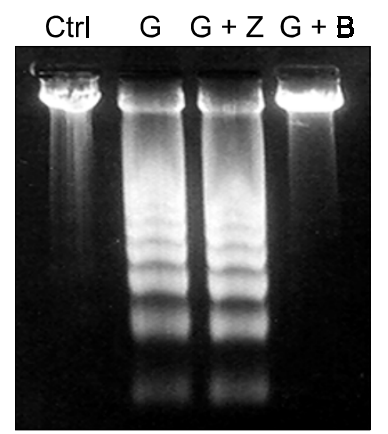

Figure 1. zVAD-fmk, unlike BocDfmk, could not protect apoptosis on genistein-treated p815 mastocytoma cells. Ctrl, control; G, genistein; Z, zVAD-fmk; B, BocDfmk. ${ }^{*} P<0.05$ vs. Ctrl. (A) Cell death quantification as determined by nuclear morphology after Hoechst staining. (B) DNA electrophoresis. 


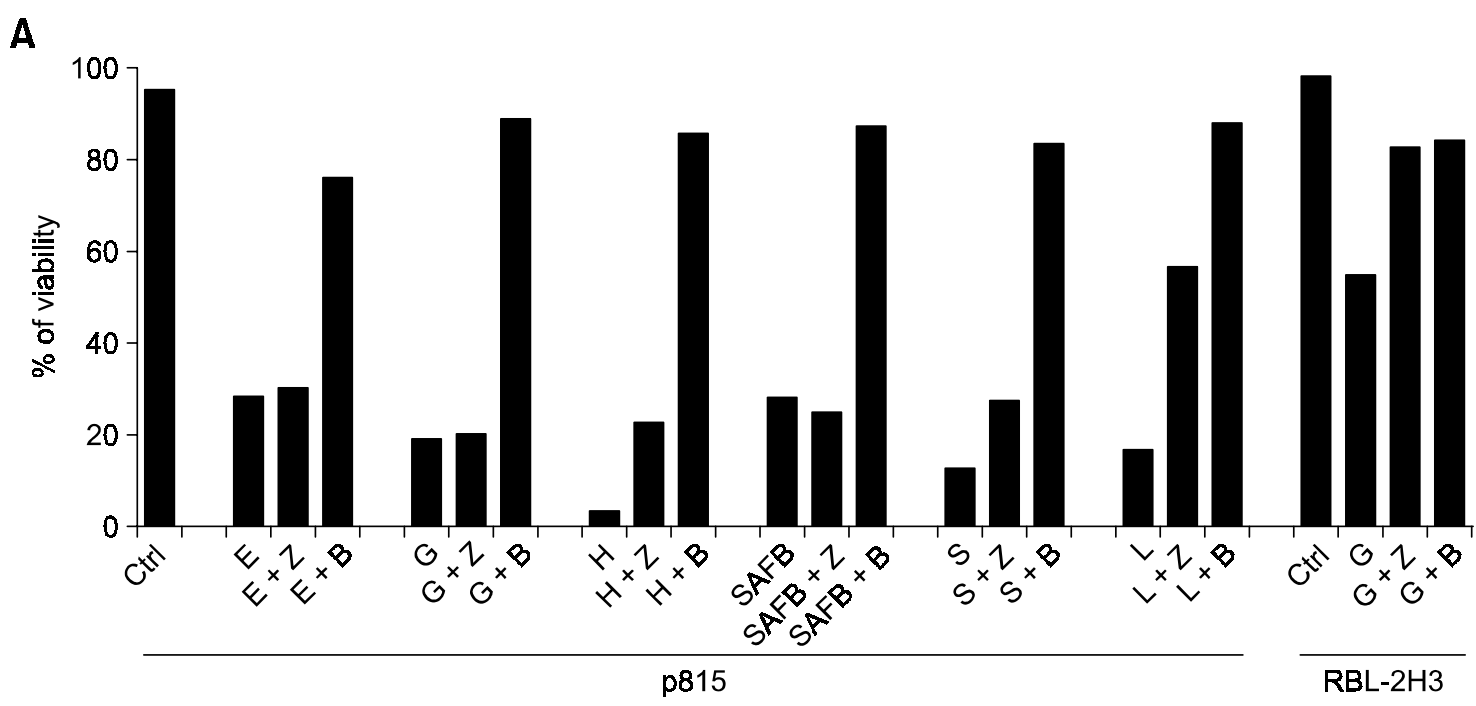

B

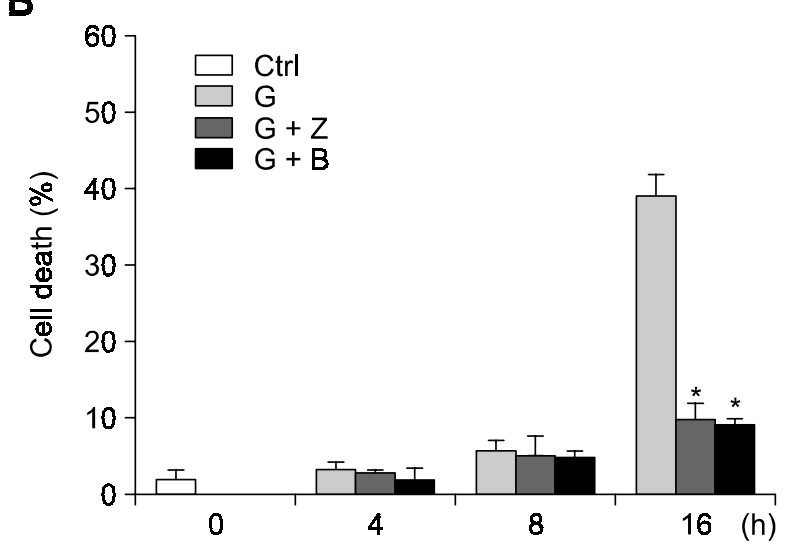

C
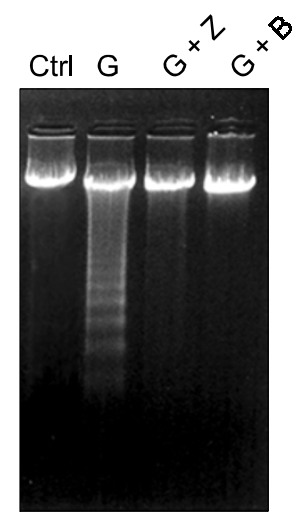

Figure 2. The incapability of zVAD-fmk in blocking apoptosis is p815 cell-selective. (A) Trypan blue exclusion assay. zVAD-fmk could not prevent apoptosis in p815 cells induced by etoposide (E) at $0.5 \mu \mathrm{g} / \mathrm{ml}$, staurosporin (S) at $0.5 \mu \mathrm{M}$ or lactacystin $(\mathrm{L})$ at 10 $\mu \mathrm{M}$. However, zVAD-fmk prevented genistein induced apoptosis in another mast cell line RBL-2H3 cells. (B) Cell death quantification of RBL-2H3 cells as determined by nuclear morphology after Hoechst staining. (C) DNA electrophoresis of RBL-2H3 cells. Ctrl, control; G, genistein; Z, ZVAD-fmk; B, BocD-fmk. ${ }^{*} P<0.05$ vs. Ctrl. A representative data from four independent experiments.

$2 \mathrm{H} 3$ (Figure $2 \mathrm{~B}$ and $\mathrm{C}$ ). These results suggest that the incapability of zVAD-fmk in preventing apoptosis is not stimulus-selective but cell-selective.

\section{BocD-fmk, compared to ZVAD-fmk, has a certain preference for inhibiting 14-3-3/Bad pathway.}

We next undertook confocal microscopy and western blot assay to seek the subcellular location from which this differential efficacy of BocD-fmk and zVAD-fmk are originated. Confocal microscopy showed that the release of mitochondrial apoptotic factors was inhibited by BocD-fmk but not by zVAD-fmk (Figure 3A). Western blot assay on several $\mathrm{Bcl}-2$ family proteins showed that tBad was produced in genistein-treated p815 cells. However, there was no detectable alteration in Bcl-2, Bax or Bak protein level after genistein treatment. The production of tBad was prevented by BocD-fmk but not by zVAD-fmk. Expectedly, neither BocD-fmk nor zVAD-fmk altered Bcl-2, Bax or Bak protein level (Figure 3B). tBAD is a more potent inducer of apoptosis than wild protein. It can induce apoptosis irrespective of the alteration of Bax, Bak, or $\mathrm{Bcl}-2$ protein and is evidently detectable only in the mitochondrial fraction (Condorelli et al., 2001). Therefore, our data suggested that the differential efficacy of zVAD-fmk and BocD-fmk was originated upstream of mitochondria.

Since BAD is sequestered in the cytosol bound to 14-3-3 under the control of survival factors, the release of Bad from 14-3-3 appears to be the 
A

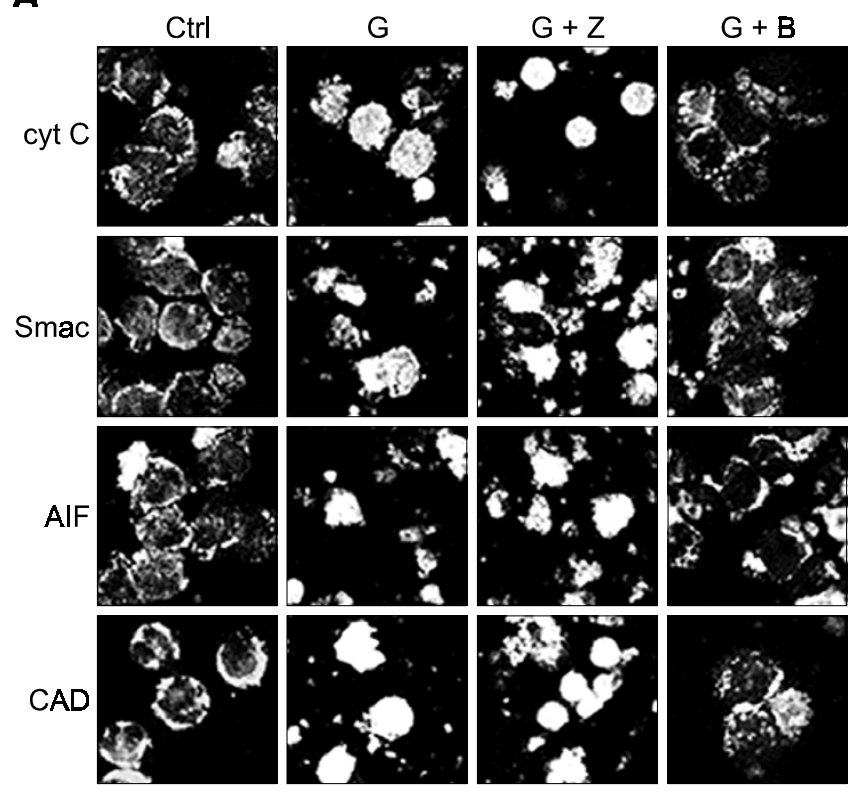

B

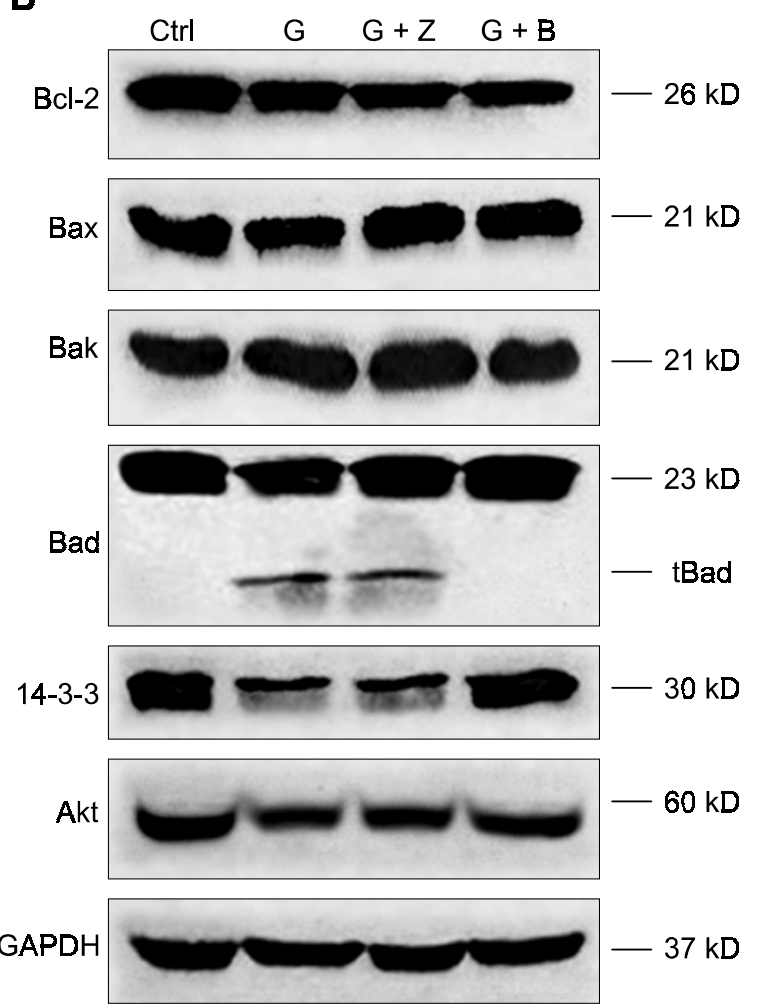

Figure 3. The differential efficacy of ZVAD-fmk and BocD-fmk was originated upstream of mitochondria. (A) Confocal microscopy of cytochrome $c$ (cyt c), Smac/Diablo (Smac), apoptosis inducing factor (AIF), and caspase-activated DNase (CAD) showing that genistein-induced release of these apoptotic factors from mitochondria was prevented by BocD-fmk but not by zVAD-fmk. Sixteen $h$ after genistein treatment. (B) Western blot assay on apoptosis-related factors. Sixteen $\mathrm{h}$ after genistein treatment. GAPDH as a loading control. zVAD-fmk, unlike BocD-fmk, could not inhibit the sequential events from 14-3-3 to Bcl-xL. Genistein-mediated downregulation of 14-3-3 and Akt was prevented by BocD-fmk but not by zVAD-fmk.

premise of the formation of tBad. Thus, we examined whether the 14-3-3 protein level was reduced in genistein-treated cells. Our data showed that 14-3-3 protein and Akt level was reduced in genistein-treated cells and that BocD-fmk but not zVAD-fmk prevented the reduction of 14-3-3 and Akt level (Figure 3B). We next undertook immunoprecipitation assay, which showed that the release of Bad from 14-3-3 in genistein treated cells was prevented by BocD-fmk but not by zVAD-fmk. It is known that phosphorylated Bad on serine residue(s) embeds in 14-3-3 consensus binding sites, and dephosphorylated Bad is released from 14-3-3 and translocated onto mitochondria. Thus, we performed immunoprecipitation assay using phosphorylation site-specific antibodies. Our data showed that phospho-ser 112- and 155-Bads were released from 14-3-3 in genistein-treated p815 cells. Noticeably, BocD-fmk but not zVAD-fmk prevented the release of phospho-ser 112- and 155-Bad from 14-3-3 (Figure 4). Bad translocated onto mitochondria is known to heterodimerize with Bcl-xL to promote the downstream apoptotic events (Zha et al., 1996). Our immunoprecipitation assay also demonstrated the production of tBad and its interaction with $\mathrm{Bcl}-\mathrm{xL}$ in genistein treated cells. Noticeably, the interaction was prevented by BocD-fmk but not by zVAD-fmk treatment (Figure 4). Aforementioned results indicate that BocD-fmk, compared to zVAD-fmk, has a certain preference for inhibiting 14-3-3/Bad pathway.

\section{Incapability of zVAD-fmk in preventing} genistein-induced apoptosis in p815 cells is primarily due to the insufficient inhibition of caspase- 6 acting on Bad/14-3-3 pathway

Our final goal was to explore whether this differential efficacy of BocD-fmk and zVAD-fmk results from the different effect in inhibiting specific caspase subtype(s). Thus we undertook caspase subtype assay to search the caspase subtype(s) involved in genistein-treated p815 cells. The assay showed that caspase- 6 and -3 were highly activated after genistein-treatment (Figure 5A). We also tested the efficacy of BocD-fmk and zVAD-fmk in preventing caspase subtypes activation, and observed that 


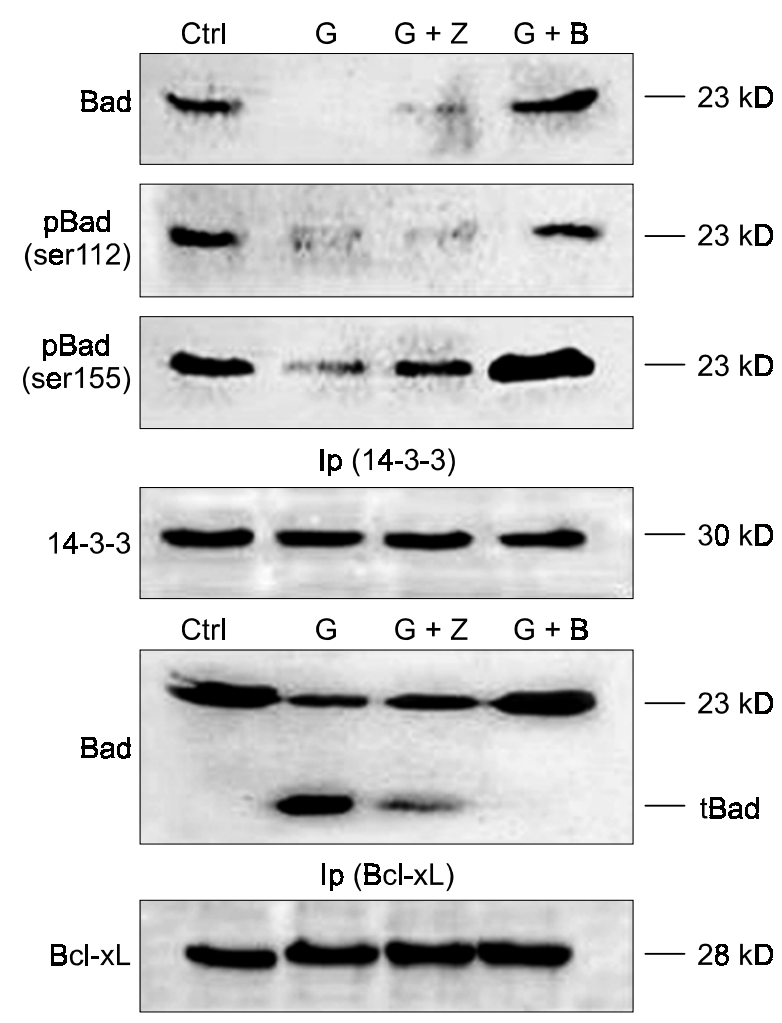

Figure 4. The reduced interaction between 14-3-3 and Bad by genistein was prevented by BocD-fmk but not by zVAD-fmk. Immnoprecipitation assay. Western blot assay using phosphorylation site-specific antibodies showed that interaction between 14-3-3 and Bad phosphorylated at Ser ${ }^{112}$ or Ser ${ }^{155}$ was decreased in genistein-treated p815 cells. The decrease of this interaction was prevented by BocD-fmk but not by zVAD-fmk. tBad was interacted with Bcl-xL in genistein-treated p815 cells. This interaction was prevented by BocD-fmk but not by zVAD-fmk.

zVAD-fmk inhibited caspase- 6 activity to a lesser degree than BocD-fmk whereas BocD-fmk and zVAD-fmk inhibited other caspase subtypes to the same degree (Figure 5A). We next asked whether co-treatment of zVAD-fmk and caspase-6 inhibitor could prevent genistein-induced apoptosis of p815 cells. Our experiment elucidated that co-treatment of zVAD-fmk and caspase- 6 specific inhibitor substantially prevented genistein-induced apoptosis. For this experiment, caspase-2 and -3 specific inhibitors were also employed since caspase-2 was known to be relatively zVAD-fmk-resistant (Ekert et al., 1999) and furthermore, caspase-2 plays a key part in premitochondrial apoptotic event and caspase-3 was observed to be highly activated in this apoptosis system. On the contrary to caspase- 6 inhibitor, co-treatment of these inhibitors with zVAD-fmk could not evidently prevent apoptosis (Figure $5 \mathrm{~B}$ ). On the other hand, caspase- 6 was not substantially activated in genistein-treated $\mathrm{RBL}-2 \mathrm{H} 3$ cells and thus we could not observe the differential efficacy of BocD-fmk and zVAD-fmk in preventing caspase-6 activation (Figure 5A).

Depending on these results, we deliberately assumed that the incapability of zVAD-fmk in preventing apoptosis is primarily due to the insufficient inhibition of caspase-6. Further, we observed that co-treatment of caspase- 6 inhibitor with zVAD-fmk prevented not only the genistein-induced downregulation of 14-3-3, Akt, and Bad but genisteininduced production of $\mathrm{tBad}$ and its interaction with $\mathrm{Bcl}-\mathrm{xL}$ (Figure 5C). Next, we examined whether zVAD-fmk and BocD-fmk showed the differential effect on 14-3-3 expression level also in apoptosis of p815 cells induced by lactacystin, etoposide, and staurosporin. zVAD-fmk, unlike BocD-fmk, also failed to prevent the reduction of 14-3-3 protein level in these types of apoptosis (Figure 6). On the other hand, we could not observe the differential efficacy of zVAD-fmk and BocD-fmk on 14-3-3 protein level in genistein-induced apoptosis of another mast cell line $\mathrm{RBL}-2 \mathrm{H}$ cells (Figure 6). Therefore, the incapability of zVAD-fmk in preventing the downregulation of 14-3-3 appears to be cell-selective phenomenon.

\section{Discussion}

Mast cells are granule-containing secretory cells that play a pivotal role in immediate-type allergic and inflammatory reactions by releasing chemical mediators such as histamine, prostaglandins, and cytokines. The mediators for degranulating mast cells have a critical function in the pathology of allergic reactions, induce tissue remodeling, and increase venular permeability and smooth muscle contraction in the bronchi and various other tissues (Hart, 2001; Bachert, 2002). The critical role of apoptosis in regulating cell numbers is now well recognized (Simon et al., 1999).

Since the number of mast cells in tissue was demonstrated to be regulated in part by apoptosis (Metcalfe et al., 1995; Park et al., 2000), induction of mast cell apoptosis may promote therapeutic efficiency for any mast cell proliferative condition. As a matter of fact, the antiinflammatory or antiallergic effect of a certain drug was demonstrated to depend on its apoptosis-inducing effect (Fuller et al., 1995; Park et al., 2001; 2002; Kim et al., 2003).

It has been known that Bad/14-3-3 pathway plays an important role in various type of apoptosis (Zha et al., 1996; Masters et al., 2001). However, its involvement in apoptosis of mast cells has not been elucidated before. We here first demonstrated that this Bad/14-3-3 pathway is involved in apoptosis of 
A
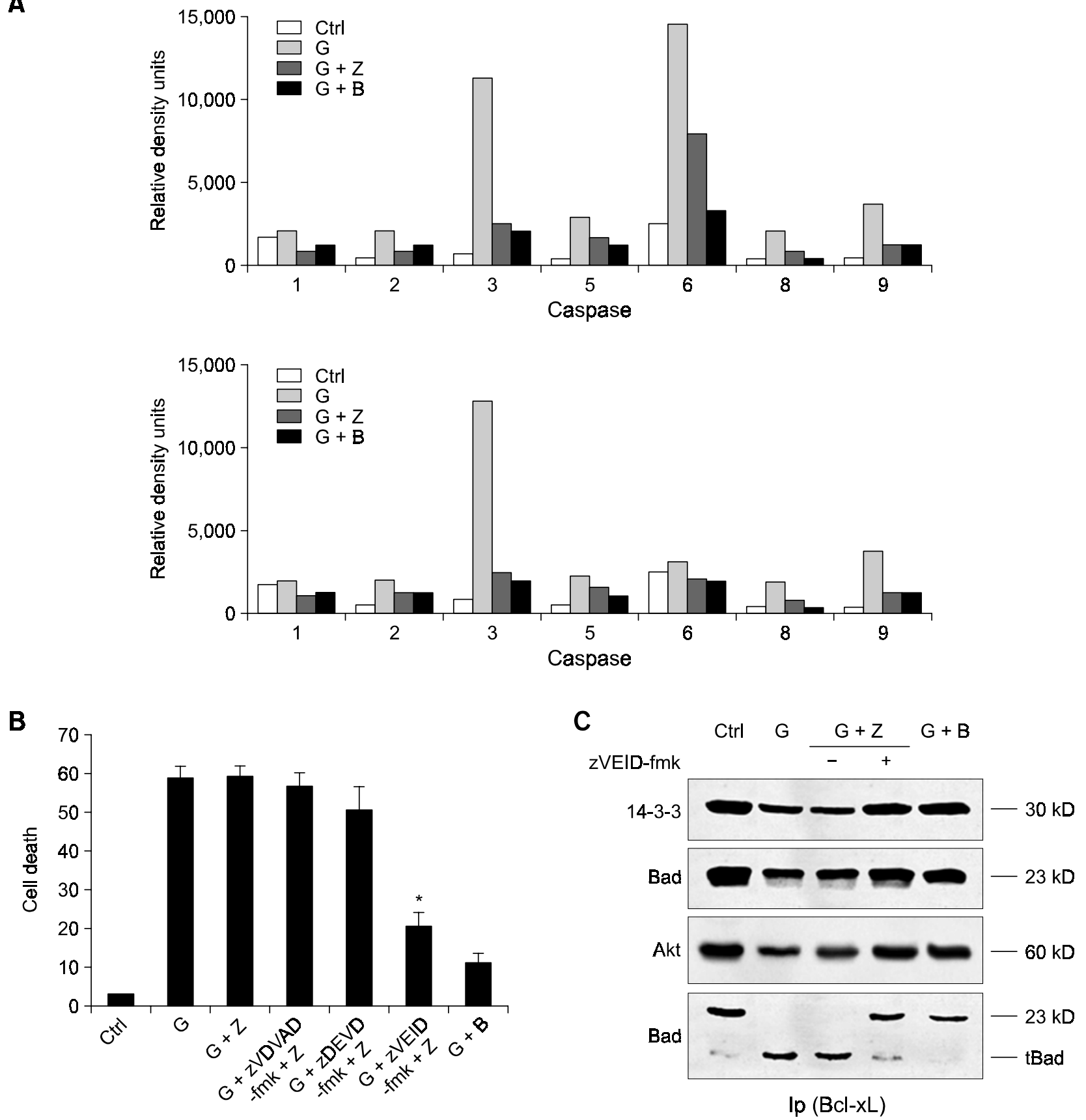

Figure 5. ZVAD-fmk, unlike BocD-fmk, could not inhibit caspase-6 acting on 14-3-3/Bad pathway. (A) Caspase activity assay on p815 cells showed that caspase-6 substrate was mostly digested (upper panel) compared to the assay on RBL-2H3 cells (lower graph). A representative data set is presented. (B) Cell death was determined by trypan blue exclusion assay. Cells co-treated with zVAD-fmk and zVEID-fmk showed the significantly decreased viability compared to zVAD-fmk-treated cells. (C) Co-treatment of ZVEID-fmk with ZVAD-fmk prevented the genistein-induced downregulation of 14-3-3, Akt, and Bad, and genistein-induced production of tBad and its interaction with Bcl-xL.

mast cells. Therefore, we assume that Bad/14-3-3 pathway could be a therapeutic target for modulating mast cell proliferating conditions.

To date, it has not been fully elucidated which type of caspase subtype is involved in 14-3-3 to Bad interaction during apoptosis. Only two elaborate studies previously reported that caspase- 3 played an important role in 14-3-3 activity. A study elicited that caspase- 3 facilitated the release of Bad from 14-3-3 and induced its interaction with $\mathrm{Bcl}-\mathrm{x}(\mathrm{L})$ (Won 


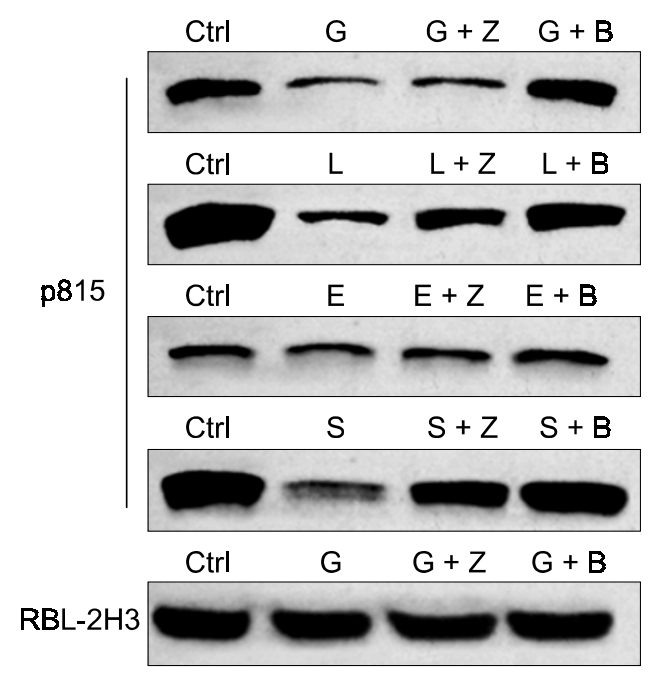

Figure 6. Several apoptosis-inducing drugs also downregulated 14-3-3 expression in p815 cells, and this downregulation was prevented by BocD-fmk but not by zVAD-fmk. G: genistein $(50 \mu \mathrm{M})$, L: lactacystin $(10 \mu \mathrm{M})$, E: etoposide $(0.5 \mu \mathrm{g} / \mathrm{ml})$, S: staurosporin $(0.5 \mu \mathrm{M})$. No alteration in 14-3-3 expression level was observed in genistein treated RBL-2H3 cells, and there was no differential efficacy of ZVAD-fmk and BocD-fmk on 14-3-3 protein level in this cell.

et al., 2003). Another study showed that caspase- 3 was involved in the release of Bax from 14-3-3 (Nomura et al., 2003). However, our data indicate that caspase-6, which plays in general as an executioner caspase, is the main caspase subtype acting on Bad/14-3-3 pathway in apoptosis of p815 cells. The involvement of casapse- 6 in 14-3-3/Bad pathway has not been documented to date. Only a recent study reported that the caspase- 6 inhibitor zVEID-fmk potently suppressed Akt ubiquitination and degradation, providing the indirect evidence of the involvement of casapse-6 in 14-3-3/Bad signalling pathway (Medina et al., 2005). In fact, it has not been fully delineated which subtype of caspases acts on each signaling event of various types of apoptosis. Therefore, our novel observations may provide further insights into understanding this issue. However, it remains also an open question through which exact molecular mechanism caspase- 6 acts on $\mathrm{Bad} / 14-3-3$.

We here raised another important issue about the efficacy of zVAD-fmk. zVAD-fmk inhibited caspase-6 activity to a lesser degree than BocD-fmk. To date, there has been no report demonstrating that zVAD-fmk at usual dose could not prevent apoptosis because of its incapability in inhibiting caspase- 6 activity and subsequent Bad/14-3-3 pathway. The understanding of caspase substrate specificities is useful in attempts to design selective inhibitors of individual caspases. Therefore, our novel observa- tion on the incapability of zVAD-fmk in preventing caspase- 6 activity is noteworthy. Our data indicate that we should be very cautious in determining the type and concentration of broad-spectrum caspase inhibitors to investigate caspase activity.

Our data also indicate that we should avoid misusing the term caspase-independent cell death (CICD). Although CICD, in the strict sense, occurs when a signal that normally engages apoptosis fails to activate caspase, the term CICD has also been used to describe the cell death which was not blocked by broad-caspase inhibitors (Talanian et al., 1997; Jones et al., 1999). However, considering that these inhibitors do not inhibit all caspases to the same extent, the term CICD should be tactfully adopted.

\section{Acknowledgement}

This work was supported by Korea Research Foundation Grant (KRF-2003-003-34-004).

\section{References}

Bachert $C$. The role of histamine in allergic disease: reappraisal of its inflammatory potential. Allergy 2002;57: 287-96

Chipuk JE, Green DR. Do inducers of apoptosis trigger caspase-independent cell death? Nat Rev Mol Cell Biol 2005; 6:268-75

Condorelli F, Salomoni P, Cotteret S, Cesi V, Srinivasula SM, Alnemri ES, Calabretta B. Caspase cleavage enhances the apoptosis-inducing effects of BAD. Mol Cell Biol 2001; 21:3025-36

Du Z, Zhang H, Gao D, Wang H, Li Y, Liu G. Antisurvivin oligonucleotides inhibit growth and induce apoptosis in human medullary thyroid carcinoma cells. Exp Mol Med 2006; 38:230-40

Ekert PG, Silke J, Vaux DL. Caspase inhibitors. Cell Death Differ 1999;6:1081-6

Fuller R, Johnson M, Bye A. Fluticasone propionate : an update on preclinical and clinical experience. Respir Med 1995; 89:3-18

Gong X, Wang M, Wu Z, Tashiro S, Onodera S, Takashi I. Pseudolaric acid $B$ induces apoptosis via activation of c-Jun $\mathrm{N}$-terminal kinase and caspase-3 in HeLa cells. Exp Mol Med 2004;36:551-6

Hart PH. Regulation of the inflammatory response in asthma by mast cell products. Immunol Cell Biol 2001;79:149-53

Holtzman MJ, Green JM, Jayaraman S, Arch RH. Regulation of $T$ cell apoptosis. Apoptosis 2000;5:459-71

Jones BE, Lo CR, Srinivasan A, Valentino KL, Czaja MJ. Ceramide induces caspase independent apoptosis in rat hepatocytes sensitized by inhibition of RNA synthesis. Hepatology 1999;30:215-22 
Kankaanranta H, Giembycz MA, Barnes PJ, Haddad el-B, Saarelainen S, Zhang X, Moilanen E, Lindsay MA. Hydrogen peroxide reverses IL-5 afforded eosinophil survival and promotes constitutive human eosinophil apoptosis. Int Arch Allergy Immunol 2002;127:73-8

Kim GC, Lee SG, Park BS, Kim JY, Song YS, Kim JM, Yoo KS, Huh GY, Jeong MH, Lim YJ, Kim HM, Yoo YH. Magnoliae flos induces apoptosis of RBL-2H3 cells via mitochondria and caspase. Int Arch Allergy Immunol 2003;131:101-10

Masters SC, Fu H. 14-3-3 proteins mediate an essential anti-apoptotic signal. J Biol Chem 2001;276:45193-200

McCarthy NJ, Whyte MK, Gilbert CS, Evan, GI. Inhibition of Ced-3/ICE-related proteases does not prevent cell death induced by oncogenes, DNA damage, or the Bcl-2 homologue Bak. J Cell Biol 1997;136:215-27

Medina EA, Afsari RR, Ravid T, Castillo SS, Erickson KL, Goldkorn T. Tumor necrosis factor-\{alpha\} decreases Akt protein levels in 3T3-L1 adipocytes via the caspase dependent ubiquitination of Akt. Endocrinology 2005;146:2726-35

Metcalfe DD, Mekori JA, Rottem M. Mast cell ontogeny and apoptosis. Exp Dermatol 1995;4:227-30

Nomura M, Shimizu S, Sugiyama T, Narita M, Ito T, Matsuda $\mathrm{H}$, Tsujimoto $\mathrm{Y}, 14-3-3$ Interacts directly with and negatively regulates pro-apoptotic Bax. J Biol Chem 2003;278:2058-65

Park BS, Kim GC, Baek SJ, Kim ND, Kim YS, Kim SK, Jeong $\mathrm{MH}, \mathrm{Lim}$ YJ, Yoo YH. Murine Bone Marrow-Derived Mast cells Exhibit Evidence of Both Apoptosis and Oncosis after IL-3 Deprivation. Immunol Invest 2000;29:51-60
Park BS, Baek SJ, Song KH, Kim KH, Jeong SJ, Jeong MH Seo SY, Lee SW, Yoo KS, Yoo YH. Genistein-induced apoptosis of p815 mastocytoma cells is mediated by Bax and augmented by a proteasome inhibitor, lactacystin. Nutr Cancer 2002;42:248-55

Park HI, Jeong MH, Lim YJ, Park BS, Kim GC, Lee YM, Kim HM, Yoo KS, Yoo YH. Syzygium aromaticum (L.) Merr. Et Perry (Myrtaceae) flower bud Induces apoptosis of p815 mastocytoma cell line. Life Sciences 2001;69:553-66

Piliponsky AM, Levi-Schaffer F. Regulation of apoptosis in mast cells. Apoptosis 2000;5:435-41

Reed JC. Apoptosis-regulating proteins as targets for drug discovery. Trends Mol Med 2001;7:314-9

Simon H, Alam R.Regulation of eosinophil apoptosis: transduction of survival and death signals. Int Arch Allergy Immunol 1999;118:7-14

Talanian RV, Yang X, Turbov J, Seth P, Ghayur T, Casiano CA, Orth K, Froelich CJ. Granule-mediated killing: pathways for granzyme B-initiated apoptosis. J Exp Med 1997;186:1323-31

Won J, Kim DY, La M, Kim D, Meadows GG, Joe CO. Cleavage of 14-3-3 protein by caspase-3 facilitates bad interaction with Bcl-x(L) during apoptosis. J Biol Chem 2003;278:19347-51

Zha J, Harada H, Yang E, Jockel J, Korsmeyer SJ. Serin phosphorylation of death agonist BAD in response to survival factor results in binding to $14-3-3$ not BCL-X(L). Cell 1996; 87:619-28

Zimmermann KC, Bonzon C, Green DR. The machinery of programmed cell death. Pharmacol Ther 2001;92:57-70 